\title{
Coordination Chemistry of Inversely Polarized Phosphaalkenes toward Group 10 Metal Centers
}

Leïla Boubekeur, ${ }^{a}$ Louis Ricard, ${ }^{\mathrm{a}}$ Pascal Le Floch, ${ }^{\mathrm{a}}$ Nicolas Mézailles, ${ }^{\mathrm{a}}$ *

\author{
${ }^{a}$ Laboratoire « Hétéroéléments et Coordination », UMR CNRS 7653 (DCPH), Département \\ de Chimie, Ecole Polytechnique, \\ 91128 Palaiseau Cédex, France \\ Tel: +33169334570 ; Fax: +3316933 3990 \\ E-mail: mezaille@poly.polytechnique.fr
}


Crystallographic data for 9' and $\mathbf{1 0}$

Figure S1: Molecular structure of 9'

Table S1-1: Crystal data and structure refinement for 9'

1 page

1 page

Table S1-2: Atomic Coordinates and equivalent isotropic displacement parameters for 9,

Table S1-3: Bond lengths $(\AA)$ and angles $\left({ }^{\circ}\right)$ for 9 '

1 page

Table S1-4: Anisotropic displacement parameters for 9'

3 pages

Table S1-5: Coordinates and equivalent isotropic

displacement parameters for 9'

1 page

Figure S2: Molecular structure of 10

1 page

Table S2-1: Crystal data and structure refinement for $\mathbf{1 0}$

1 page

1 page

Table S2-2: Atomic Coordinates and equivalent isotropic

1 page

displacement parameters for $\mathbf{1 0}$

Table S2-3: Bond lengths $(\AA)$ and angles $\left(^{\circ}\right)$ for 10

2 pages

Table S2-4: Anisotropic displacement parameters for 10

1 page

Table S2-5: Coordinates and equivalent isotropic

displacement parameters for $\mathbf{1 0}$

1 page 
Figure S1: Molecular structure of 9'

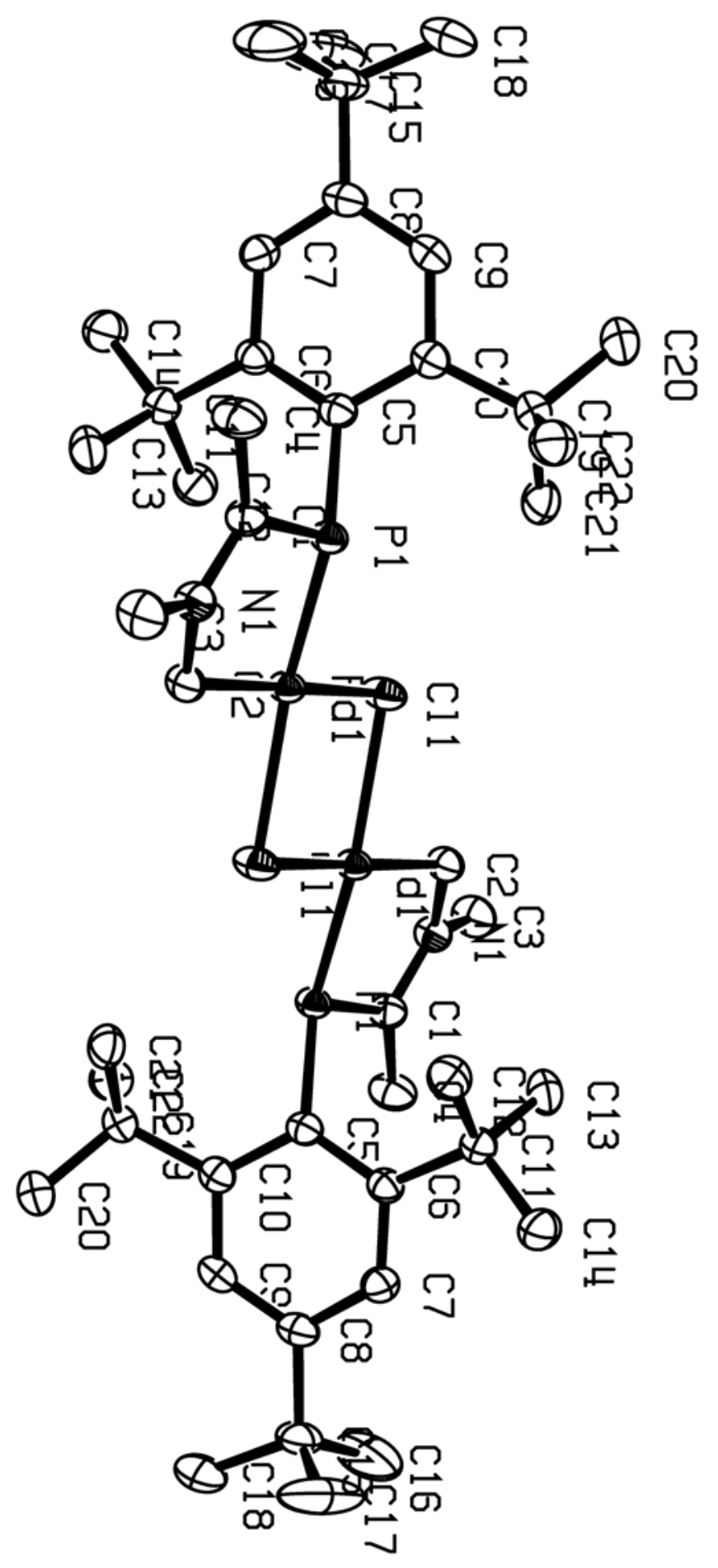


Table S1-1. Crystal data for 9'

\begin{tabular}{|c|c|}
\hline Compound & pdinvalc \\
\hline Molecular formula & $\mathrm{C}_{44} \mathrm{H}_{74} \mathrm{Cl}_{2} \mathrm{~N}_{2} \mathrm{P}_{2} \mathrm{Pd}_{2}, 2\left(\mathrm{C}_{6} \mathrm{D}_{6}\right)$ \\
\hline Molecular weight & 1144.97 \\
\hline Crystal habit & yellow plate \\
\hline Crystal dimensions $(\mathrm{mm})$ & $0.20 \times 0.18 \times 0.12$ \\
\hline Crystal system & monoclinic \\
\hline Space group & $\mathrm{C} 2 / \mathrm{c}$ \\
\hline $\mathrm{a}(\AA)$ & $36.0050(10)$ \\
\hline $\mathrm{b}(\AA)$ & $9.8830(10)$ \\
\hline$c(\AA)$ & $17.7840(10)$ \\
\hline$\alpha\left(^{\circ}\right)$ & 90.00 \\
\hline$\beta\left(^{\circ}\right)$ & $117.5200(10)$ \\
\hline$\gamma\left({ }^{\circ}\right)$ & 90.00 \\
\hline $\mathrm{V}\left(\AA^{3}\right)$ & $5612.2(7)$ \\
\hline $\mathrm{Z}$ & 4 \\
\hline $\mathrm{d}\left(\mathrm{g}-\mathrm{cm}^{-3}\right)$ & 1.355 \\
\hline $\mathrm{F}(000)$ & 2368 \\
\hline$\mu\left(\mathrm{cm}^{-1}\right)$ & 0.829 \\
\hline Absorption corrections & multi-scan ; $0.8517 \min , 0.9071 \max$ \\
\hline Diffractometer & KappaCCD \\
\hline X-ray source & $\mathrm{MoK} \alpha$ \\
\hline$\lambda(\AA)$ & 0.71069 \\
\hline Monochromator & graphite \\
\hline $\mathrm{T}(\mathrm{K})$ & $150.0(1)$ \\
\hline Scan mode & phi and omega scans \\
\hline Maximum $\theta$ & 30.03 \\
\hline HKL ranges & $-5050 ;-1313 ;-2524$ \\
\hline Reflections measured & 15209 \\
\hline Unique data & 8167 \\
\hline Rint & 0.0259 \\
\hline Reflections used & 6368 \\
\hline Criterion & $\mathrm{I}>2 \sigma \mathrm{I})$ \\
\hline Refinement type & Fsqd \\
\hline Hydrogen atoms & mixed \\
\hline Parameters refined & 332 \\
\hline Reflections / parameter & 19 \\
\hline wR2 & 0.0912 \\
\hline $\mathrm{R} 1$ & 0.0334 \\
\hline Weights $a, b$ & $0.0460 ; 1.0259$ \\
\hline GoF & 1.061 \\
\hline difference peak / hole $\left(\mathrm{e} \AA^{-3}\right)$ & $1.018(.080) /-.794(.080)$ \\
\hline
\end{tabular}


Table S1-2. Atomic Coordinates (A $\times 10^{\wedge} 4$ ) and equivalent isotropic displacement parameters $\left(A^{\wedge} 2 \times 10^{\wedge} 3\right)$ for $\mathbf{9}^{\prime}$

\begin{tabular}{|c|c|c|c|c|}
\hline atom & $\mathrm{x}$ & $\mathrm{y}$ & $\mathrm{z}$ & $\mathrm{U}(\mathrm{eq})$ \\
\hline $\mathrm{Pd}(1)$ & $0(10000)$ & $1316(1)$ & $4230(1)$ & $22(1)$ \\
\hline $\mathrm{Cl}(1)$ & $2760(1)$ & $1208(1)$ & $5644(1)$ & $30(1)$ \\
\hline$P(1)$ & $1553(1)$ & $1079(1)$ & $3098(1)$ & $23(1)$ \\
\hline $\mathrm{N}(1)$ & $1804(1)$ & $-1390(2)$ & $3516(1)$ & $27(1)$ \\
\hline$C(1)$ & $1514(1)$ & $-657(2)$ & $2894(1)$ & $26(1)$ \\
\hline$C(2)$ & $2188(1)$ & $-710(2)$ & $4108(1)$ & $26(1)$ \\
\hline$C(3)$ & $1767(1)$ & $-2851(2)$ & $3615(2)$ & $36(1)$ \\
\hline$C(4)$ & $1164(1)$ & $-1344(2)$ & $2156(2)$ & $37(1)$ \\
\hline$C(5)$ & $1298(1)$ & $1805(2)$ & $2016(1)$ & $23(1)$ \\
\hline$C(6)$ & $1438(1)$ & $1559(2)$ & $1398(1)$ & $24(1)$ \\
\hline$C(7)$ & $1147(1)$ & $1628(2)$ & $547(1)$ & $28(1)$ \\
\hline$C(8)$ & $730(1)$ & $2000(2)$ & $273(1)$ & $29(1)$ \\
\hline$C(9)$ & $620(1)$ & $2428(2)$ & $881(1)$ & $27(1)$ \\
\hline$C(10)$ & $895(1)$ & $2376(2)$ & $1752(1)$ & $25(1)$ \\
\hline$C(11)$ & $1904(1)$ & $1330(2)$ & $1606(1)$ & $27(1)$ \\
\hline$C(12)$ & $2179(1)$ & $2396(2)$ & $2248(2)$ & $35(1)$ \\
\hline$C(13)$ & $2067(1)$ & $-96(2)$ & $1941(2)$ & $36(1)$ \\
\hline C (14) & $1966(1)$ & $1526(3)$ & $816(2)$ & $39(1)$ \\
\hline$C(15)$ & $420(1)$ & $1994(3)$ & $-680(1)$ & $37(1)$ \\
\hline$C(16)$ & $373(2)$ & $595(5)$ & $-1015(3)$ & $94(2)$ \\
\hline$C(17)$ & $577(1)$ & $2999(7)$ & $-1134(2)$ & $84(2)$ \\
\hline$C(18)$ & $-16(1)$ & $2523(5)$ & $-850(2)$ & $62(1)$ \\
\hline$C(16 A)$ & $123(6)$ & $790(20)$ & $-810(10)$ & $94(2)$ \\
\hline$C(17 A)$ & $651(5)$ & $1660(20)$ & $-1240(10)$ & $84(2)$ \\
\hline$C(18 A)$ & $183(4)$ & $3220(20)$ & $-950(8)$ & $62(1)$ \\
\hline$C(19)$ & $741(1)$ & $2989(2)$ & $2360(1)$ & $29(1)$ \\
\hline$C(20)$ & $372(1)$ & $3971(3)$ & $1889(2)$ & $44(1)$ \\
\hline$C(21)$ & $1086(1)$ & $3838(2)$ & $3053(2)$ & $34(1)$ \\
\hline$C(22)$ & $581(1)$ & $1876(3)$ & $2748(1)$ & $37(1)$ \\
\hline$C(23)$ & $903(1)$ & $7094(3)$ & $85(2)$ & $58(1)$ \\
\hline$D(23)$ & $600(10)$ & $7500(30)$ & $-240(20)$ & 69 \\
\hline$C(24)$ & $1254(1)$ & $7640(3)$ & $72(2)$ & $58(1)$ \\
\hline D (24) & $1210(10)$ & $8490(30)$ & $-280(20)$ & 69 \\
\hline$C(25)$ & $1635(1)$ & $7015(3)$ & $518(2)$ & $59(1)$ \\
\hline$D(25)$ & $1890(10)$ & $7430(30)$ & $470(20)$ & 71 \\
\hline$C(26)$ & $1664(1)$ & $5861(4)$ & $970(3)$ & $72(1)$ \\
\hline$D(26)$ & $1910(10)$ & $5370(40)$ & $1260(20)$ & 86 \\
\hline$C(27)$ & $1314(1)$ & $5335(3)$ & $985(3)$ & $68(1)$ \\
\hline$D(27)$ & $1340(10)$ & $4530(40)$ & $1290(20)$ & 82 \\
\hline $\mathrm{C}(28)$ & $931(1)$ & $5953(3)$ & $525(2)$ & $57(1)$ \\
\hline $\mathrm{D}(28)$ & $670(10)$ & $5590(30)$ & $490(20)$ & 69 \\
\hline
\end{tabular}

U(eq) is defined as $1 / 3$ the trace of the Uij tensor. 
Table S1-3. Bond lengths (A) and angles (deg) for 9'

\begin{tabular}{|c|c|c|c|}
\hline $\mathrm{Pd}(1)-\mathrm{C}(2)$ & $2.018(2)$ & $\mathrm{Pd}(1)-\mathrm{P}(1)$ & $2.2177(5)$ \\
\hline $\mathrm{Pd}(1)-\mathrm{Cl}(1)$ & $2.4293(5$ & $\mathrm{Pd}(1)-\mathrm{Cl}(1) \# 7$ & $2.4591(6)$ \\
\hline $\mathrm{Cl}(1)-\mathrm{Pd}(1)$ \# 7 & $2.4592(6$ & $P(1)-C(1)$ & $1.746(2)$ \\
\hline$P(1)-C(5)$ & $1.852(2)$ & $\mathrm{N}(1)-\mathrm{C}(1)$ & $1.331(2)$ \\
\hline$N(1)-C(2)$ & $1.457(2)$ & $N(1)-C(3)$ & $1.468(3)$ \\
\hline$C(1)-C(4)$ & $1.498(3)$ & $\mathrm{C}(2)-\mathrm{H}(2 \mathrm{~A})$ & 0.9900 \\
\hline $\mathrm{C}(2)-\mathrm{H}(2 \mathrm{~B})$ & 0.9900 & $C(3)-H(3 A)$ & 0.9800 \\
\hline$C(3)-H(3 B)$ & 0.9800 & $\mathrm{C}(3)-\mathrm{H}(3 \mathrm{C})$ & 0.9800 \\
\hline $\mathrm{C}(4)-\mathrm{H}(4 \mathrm{~A})$ & 0.9800 & $\mathrm{C}(4)-\mathrm{H}(4 \mathrm{~B})$ & 0.9800 \\
\hline $\mathrm{C}(4)-\mathrm{H}(4 \mathrm{C})$ & 0.9800 & $C(5)-C(10)$ & $1.420(3)$ \\
\hline$C(5)-C(6)$ & $1.425(3)$ & $C(6)-C(7)$ & $1.389(3)$ \\
\hline$C(6)-C(11)$ & $1.559(3)$ & $C(7)-C(8)$ & $1.395(3)$ \\
\hline $\mathrm{C}(7)-\mathrm{H}(7)$ & 0.9500 & $C(8)-C(9)$ & $1.378(3)$ \\
\hline$C(8)-C(15)$ & $1.540(3)$ & $C(9)-C(10)$ & $1.401(3)$ \\
\hline $\mathrm{C}(9)-\mathrm{H}(9)$ & 0.9500 & $C(10)-C(19)$ & $1.551(3)$ \\
\hline$C(11)-C(14)$ & $1.532(3)$ & $C(11)-C(12)$ & $1.532(3)$ \\
\hline$C(11)-C(13)$ & $1.536(3)$ & $\mathrm{C}(12)-\mathrm{H}(12 \mathrm{~A})$ & 0.9800 \\
\hline $\mathrm{C}(12)-\mathrm{H}(12 \mathrm{~B})$ & 0.9800 & $\mathrm{C}(12)-\mathrm{H}(12 \mathrm{C})$ & 0.9800 \\
\hline $\mathrm{C}(13)-\mathrm{H}(13 \mathrm{~A})$ & 0.9800 & $\mathrm{C}(13)-\mathrm{H}(13 \mathrm{~B})$ & 0.9800 \\
\hline$C(13)-H(13 C)$ & 0.9800 & $\mathrm{C}(14)-\mathrm{H}(14 \mathrm{~A})$ & 0.9800 \\
\hline $\mathrm{C}(14)-\mathrm{H}(14 \mathrm{~B})$ & 0.9800 & $\mathrm{C}(14)-\mathrm{H}(14 \mathrm{C})$ & 0.9800 \\
\hline$C(15)-C(18 A)$ & $1.44(2)$ & $C(15)-C(16)$ & $1.483(5)$ \\
\hline$C(15)-C(17)$ & $1.541(5)$ & $C(15)-C(16 A)$ & $1.54(2)$ \\
\hline C (15) -C (18) & $1.547(4)$ & $C(15)-C(17 A)$ & $1.60(2)$ \\
\hline $\mathrm{C}(16)-\mathrm{H}(16 \mathrm{~A})$ & 0.9800 & $\mathrm{C}(16)-\mathrm{H}(16 \mathrm{~B})$ & 0.9800 \\
\hline $\mathrm{C}(16)-\mathrm{H}(16 \mathrm{C})$ & 0.9800 & $\mathrm{C}(17)-\mathrm{H}(17 \mathrm{~A})$ & 0.9800 \\
\hline $\mathrm{C}(17)-\mathrm{H}(17 \mathrm{~B})$ & 0.9800 & $\mathrm{C}(17)-\mathrm{H}(17 \mathrm{C})$ & 0.9800 \\
\hline $\mathrm{C}(18)-\mathrm{H}(18 \mathrm{~A})$ & 0.9800 & $\mathrm{C}(18)-\mathrm{H}(18 \mathrm{~B})$ & 0.9800 \\
\hline $\mathrm{C}(18)-\mathrm{H}(18 \mathrm{C})$ & 0.9800 & $C(16 A)-H(16 D)$ & 0.9800 \\
\hline $\mathrm{C}(16 \mathrm{~A})-\mathrm{H}(16 \mathrm{E})$ & 0.9800 & $\mathrm{C}(16 \mathrm{~A})-\mathrm{H}(16 \mathrm{~F})$ & 0.9800 \\
\hline $\mathrm{C}(17 \mathrm{~A})-\mathrm{H}(17 \mathrm{D})$ & 0.9800 & $\mathrm{C}(17 \mathrm{~A})-\mathrm{H}(17 \mathrm{E})$ & 0.9800 \\
\hline $\mathrm{C}(17 \mathrm{~A})-\mathrm{H}(17 \mathrm{~F})$ & 0.9800 & $\mathrm{C}(18 \mathrm{~A})-\mathrm{H}(18 \mathrm{D})$ & 0.9800 \\
\hline $\mathrm{C}(18 \mathrm{~A})-\mathrm{H}(18 \mathrm{E})$ & 0.9800 & $\mathrm{C}(18 \mathrm{~A})-\mathrm{H}(18 \mathrm{~F})$ & 0.9800 \\
\hline$C(19)-C(21)$ & $1.535(3)$ & $C(19)-C(20)$ & $1.541(3)$ \\
\hline$C(19)-C(22)$ & $1.544(3)$ & $\mathrm{C}(20)-\mathrm{H}(20 \mathrm{~A})$ & 0.9800 \\
\hline $\mathrm{C}(20)-\mathrm{H}(20 \mathrm{~B})$ & 0.9800 & $\mathrm{C}(20)-\mathrm{H}(20 \mathrm{C})$ & 0.9800 \\
\hline $\mathrm{C}(21)-\mathrm{H}(21 \mathrm{~A})$ & 0.9800 & $\mathrm{C}(21)-\mathrm{H}(21 \mathrm{~B})$ & 0.9800 \\
\hline $\mathrm{C}(21)-\mathrm{H}(21 \mathrm{C})$ & 0.9800 & $\mathrm{C}(22)-\mathrm{H}(22 \mathrm{~A})$ & 0.9800 \\
\hline $\mathrm{C}(22)-\mathrm{H}(22 \mathrm{~B})$ & 0.9800 & $\mathrm{C}(22)-\mathrm{H}(22 \mathrm{C})$ & 0.9800 \\
\hline C (23) -C (28) & $1.350(4)$ & $C(23)-C(24)$ & $1.385(4)$ \\
\hline$C(23)-D(23)$ & $1.05(3)$ & $C(24)-C(25)$ & $1.373(4)$ \\
\hline$C(24)-D(24)$ & $1.02(3)$ & $C(25)-C(26)$ & $1.371(5)$ \\
\hline$C(25)-D(25)$ & $1.07(3)$ & $C(26)-C(27)$ & $1.374(5)$ \\
\hline$C(26)-D(26)$ & $0.94(3)$ & $C(27)-C(28)$ & $1.380(4)$ \\
\hline C (27) -D (27) & $0.95(4)$ & $\mathrm{C}(28)-\mathrm{D}(28)$ & $0.99(3)$ \\
\hline
\end{tabular}

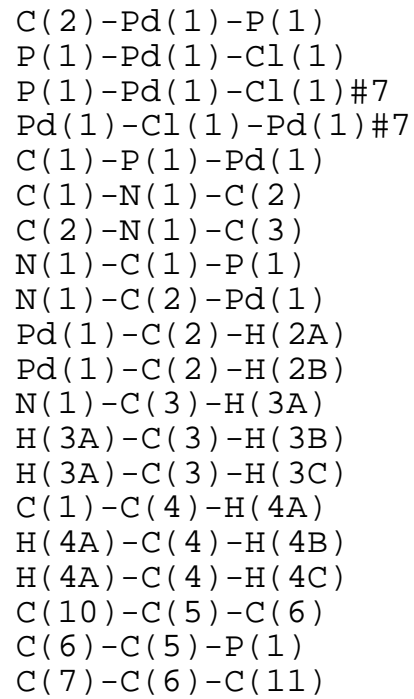

$82.21(5)$ $164.69(2)$ $101.82(2)$ $93.07(2)$ $104.07(7)$ $117.8(2)$ $118.4(2)$ $113.9(1)$ $118.0(1)$ 107.8 107.8 109.5 109.5 109.5 109.5 109.5 109.5 $119.0(2)$ $123.6(1)$ $117.1(2)$
$\mathrm{C}(2)-\mathrm{Pd}(1)-\mathrm{Cl}(1)$ $\mathrm{C}(2)-\mathrm{Pd}(1)-\mathrm{Cl}(1) \# 7$ $\mathrm{Cl}(1)-\mathrm{Pd}(1)-\mathrm{Cl}(1)$ \# 7 $\mathrm{C}(1)-\mathrm{P}(1)-\mathrm{C}(5)$ $\mathrm{C}(5)-\mathrm{P}(1)-\mathrm{Pd}(1)$ $\mathrm{C}(1)-\mathrm{N}(1)-\mathrm{C}(3)$ $\mathrm{N}(1)-\mathrm{C}(1)-\mathrm{C}(4)$ $\mathrm{C}(4)-\mathrm{C}(1)-\mathrm{P}(1)$ $\mathrm{N}(1)-\mathrm{C}(2)-\mathrm{H}(2 \mathrm{~A})$ $\mathrm{N}(1)-\mathrm{C}(2)-\mathrm{H}(2 \mathrm{~B})$ $\mathrm{H}(2 \mathrm{~A})-\mathrm{C}(2)-\mathrm{H}(2 \mathrm{~B})$ $\mathrm{N}(1)-\mathrm{C}(3)-\mathrm{H}(3 \mathrm{~B})$ $\mathrm{N}(1)-\mathrm{C}(3)-\mathrm{H}(3 \mathrm{C})$ $\mathrm{H}(3 \mathrm{~B})-\mathrm{C}(3)-\mathrm{H}(3 \mathrm{C})$ $\mathrm{C}(1)-\mathrm{C}(4)-\mathrm{H}(4 \mathrm{~B})$ $\mathrm{C}(1)-\mathrm{C}(4)-\mathrm{H}(4 \mathrm{C})$ $\mathrm{H}(4 \mathrm{~B})-\mathrm{C}(4)-\mathrm{H}(4 \mathrm{C})$ $\mathrm{C}(10)-\mathrm{C}(5)-\mathrm{P}(1)$ $C(7)-C(6)-C(5)$ $C(5)-C(6)-C(11)$
$90.73(5)$ $171.96(6)$ $86.93(2)$ $102.2(1)$ $135.27(6)$ 123.7 (2) $120.1(2)$ $125.6(2)$ 107.8 107.8 107.1 109.5 109.5 109.5 109.5 109.5 109.5 $116.0(2)$ 118.2 (2) $124.5(2)$ 
$122.9(2)$

118.6

$122.8(2)$

$122.9(2)$

118.5

$117.3(2)$

$106.2(2)$

$110.1(2)$

$109.3(2)$

109.5

109.5

109.5

109.5

109.5

109.5

109.5

109.5

109.5

$135.8(6)$

$109.6(2)$

$113.1(4)$

$110(1)$

$105.6(6)$

$41.8(7)$

$111.5(2)$

$70.3(7)$

$63.5(8)$

$52.1(8)$

$136.1(6)$

109.5

109.5

109.5

109.5

109.5

109.5

109.5

109.5

109.5

109.5

109.5

109.5

109.5

$105.5(2)$

$106.7(2)$

$111.3(2)$

109.5

109.5

109.5

109.5

109.5

109.5

109.5

109.5

109.5

$120.7(3)$

$125(2)$

$123(2)$

$119.9(3)$

$117(2)$

$123(2)$

$119.7(3)$

$121(2)$

$117(2)$
$\mathrm{C}(6)-\mathrm{C}(7)-\mathrm{H}(7)$

$C(9)-C(8)-C(7)$

$\mathrm{C}(7)-\mathrm{C}(8)-\mathrm{C}(15)$

$\mathrm{C}(8)-\mathrm{C}(9)-\mathrm{H}(9)$

$C(9)-C(10)-C(5)$

C (5) $-\mathrm{C}(10)-\mathrm{C}(19)$

C (14) -C (11) -C (13)

$C(14)-C(11)-C(6)$

$C(13)-C(11)-C(6)$

$\mathrm{C}(11)-\mathrm{C}(12)-\mathrm{H}(12 \mathrm{~B})$

$\mathrm{C}(11)-\mathrm{C}(12)-\mathrm{H}(12 \mathrm{C})$

$\mathrm{H}(12 \mathrm{~B})-\mathrm{C}(12)-\mathrm{H}(12 \mathrm{C})$

$\mathrm{C}(11)-\mathrm{C}(13)-\mathrm{H}(13 \mathrm{~B})$

$\mathrm{C}(11)-\mathrm{C}(13)-\mathrm{H}(13 \mathrm{C})$

$\mathrm{H}(13 \mathrm{~B})-\mathrm{C}(13)-\mathrm{H}(13 \mathrm{C})$

$\mathrm{C}(11)-\mathrm{C}(14)-\mathrm{H}(14 \mathrm{~B})$

$\mathrm{C}(11)-\mathrm{C}(14)-\mathrm{H}(14 \mathrm{C})$

$\mathrm{H}(14 \mathrm{~B})-\mathrm{C}(14)-\mathrm{H}(14 \mathrm{C})$

$\mathrm{C}(18 \mathrm{~A})-\mathrm{C}(15)-\mathrm{C}(8)$

$C(18 A)-C(15)-C(17)$

$C(8)-C(15)-C(17)$

$C(16)-C(15)-C(16 A)$

$C(17)-C(15)-C(16 A)$

$C(16)-C(15)-C(18)$

$C(17)-C(15)-C(18)$

$C(18 A)-C(15)-C(17 A)$

$C(8)-C(15)-C(17 A)$

$C(16 A)-C(15)-C(17 A)$

$\mathrm{C}(15)-\mathrm{C}(16)-\mathrm{H}(16 \mathrm{~A})$

$\mathrm{C}(15)-\mathrm{C}(16)-\mathrm{H}(16 \mathrm{C})$

$\mathrm{C}(15)-\mathrm{C}(17)-\mathrm{H}(17 \mathrm{~B})$

$\mathrm{C}(15)-\mathrm{C}(18)-\mathrm{H}(18 \mathrm{~A})$

$\mathrm{C}(15)-\mathrm{C}(18)-\mathrm{H}(18 \mathrm{C})$

$\mathrm{C}(15)-\mathrm{C}(16 \mathrm{~A})-\mathrm{H}(16 \mathrm{E})$

$\mathrm{C}(15)-\mathrm{C}(16 \mathrm{~A})-\mathrm{H}(16 \mathrm{~F})$

$\mathrm{H}(16 \mathrm{E})-\mathrm{C}(16 \mathrm{~A})-\mathrm{H}(16 \mathrm{~F})$

$\mathrm{C}(15)-\mathrm{C}(17 \mathrm{~A})-\mathrm{H}(17 \mathrm{E})$

$\mathrm{C}(15)-\mathrm{C}(17 \mathrm{~A})-\mathrm{H}(17 \mathrm{~F})$

$\mathrm{H}(17 \mathrm{E})-\mathrm{C}(17 \mathrm{~A})-\mathrm{H}(17 \mathrm{~F})$

$\mathrm{C}(15)-\mathrm{C}(18 \mathrm{~A})-\mathrm{H}(18 \mathrm{E})$

$\mathrm{C}(15)-\mathrm{C}(18 \mathrm{~A})-\mathrm{H}(18 \mathrm{~F})$

$\mathrm{H}(18 \mathrm{E})-\mathrm{C}(18 \mathrm{~A})-\mathrm{H}(18 \mathrm{~F})$

$C(21)-C(19)-C(22)$

$C(21)-C(19)-C(10)$

$C(22)-C(19)-C(10)$

$\mathrm{C}(19)-\mathrm{C}(20)-\mathrm{H}(20 \mathrm{~B})$

$\mathrm{C}(19)-\mathrm{C}(20)-\mathrm{H}(20 \mathrm{C})$

$\mathrm{H}(20 \mathrm{~B})-\mathrm{C}(20)-\mathrm{H}(20 \mathrm{C})$

$\mathrm{C}(19)-\mathrm{C}(21)-\mathrm{H}(21 \mathrm{~B})$

$\mathrm{C}(19)-\mathrm{C}(21)-\mathrm{H}(21 \mathrm{C})$

$\mathrm{H}(21 \mathrm{~B})-\mathrm{C}(21)-\mathrm{H}(21 \mathrm{C})$

$\mathrm{C}(19)-\mathrm{C}(22)-\mathrm{H}(22 \mathrm{~B})$

$\mathrm{C}(19)-\mathrm{C}(22)-\mathrm{H}(22 \mathrm{C})$

$\mathrm{H}(22 \mathrm{~B})-\mathrm{C}(22)-\mathrm{H}(22 \mathrm{C})$

$\mathrm{C}(28)-\mathrm{C}(23)-\mathrm{D}(23)$

C (25) $-\mathrm{C}(24)-\mathrm{C}(23)$

$C(23)-C(24)-D(24)$

$\mathrm{C}(26)-\mathrm{C}(25)-\mathrm{D}(25)$

C (25) $-\mathrm{C}(26)-\mathrm{C}(27)$

$\mathrm{C}(27)-\mathrm{C}(26)-\mathrm{D}(26)$

C (26) $-\mathrm{C}(27)-\mathrm{D}(27)$

$\mathrm{C}(23)-\mathrm{C}(28)-\mathrm{C}(27)$

$\mathrm{C}(27)-\mathrm{C}(28)-\mathrm{D}(28)$
118.6

$117.3(2)$

$119.9(2)$

118.5

$118.2(2)$

$124.5(2)$

$106.3(2)$

$111.0(2)$

$113.7(2)$

109.5

109.5

109.5

109.5

109.5

109.5

109.5

109.5

109.5

$112.4(6)$

$65.4(8)$

$108.6(2)$

$43.8(7)$

$144.4(7)$

$108.7(3)$

$105.3(3)$

$112(1)$

$111.6(5)$

$106(1)$

109.5

109.5

109.5

109.5

109.5

109.5

109.5

109.5

109.5

109.5

109.5

109.5

109.5

109.5

$111.2(2)$

$110.8(2)$

$111.2(2)$

109.5

109.5

109.5

109.5

109.5

109.5

109.5

109.5

109.5

115 (2)

$119.4(3)$

$118(2)$

$123(2)$

$120.3(3)$

$117(2)$

$120(2)$

$120.0(3)$

$123(2)$

Estimated standard deviations are given in the parenthesis. Symmetry operators : :

$$
\begin{array}{lll}
1: x, y, z & 2:-x, y,-z+1 / 2 & 3: x+1 / 2, y+1 / 2, z \\
4:-x+1 / 2, y+1 / 2,-z+1 / & 5:-x,-y,-z & 6: x,-y, z-1 / 2 \\
7:-x+1 / 2,-y+1 / 2,-z & 8: x+1 / 2,-y+1 / 2, z-1 / 2 &
\end{array}
$$


Table S1-4. Anisotropic displacement parameters (A^2 x 10^3) for 9'

\begin{tabular}{|c|c|c|c|c|c|c|}
\hline atom & $\mathrm{U} 11$ & $\mathrm{U} 22$ & U33 & U23 & U13 & U12 \\
\hline $\mathrm{Pd}(1)$ & $22(1)$ & $19(1)$ & $19(1)$ & $-1(1)$ & $5(1)$ & $-1(1)$ \\
\hline $\mathrm{Cl}(1)$ & $30(1)$ & $21(1)$ & $24(1)$ & $0(1)$ & $1(1)$ & $-1(1)$ \\
\hline$P(1)$ & $21(1)$ & $21(1)$ & $20(1)$ & $-1(1)$ & $5(1)$ & $0(1)$ \\
\hline $\mathrm{N}(1)$ & $27(1)$ & $21(1)$ & $28(1)$ & $-2(1)$ & $9(1)$ & $-2(1)$ \\
\hline$C(1)$ & $26(1)$ & $25(1)$ & $25(1)$ & $-1(1)$ & $10(1)$ & $-3(1)$ \\
\hline$C(2)$ & $26(1)$ & $20(1)$ & $26(1)$ & $-1(1)$ & $8(1)$ & $1(1)$ \\
\hline$C(3)$ & $41(1)$ & $20(1)$ & $41(1)$ & $1(1)$ & $14(1)$ & $-2(1)$ \\
\hline C (4) & 35 (1) & $29(1)$ & $32(1)$ & $-4(1)$ & $4(1)$ & $-6(1)$ \\
\hline$C(5)$ & $23(1)$ & $20(1)$ & $22(1)$ & $-1(1)$ & $7(1)$ & $-1(1)$ \\
\hline$C(6)$ & $23(1)$ & $23(1)$ & $24(1)$ & $-1(1)$ & $8(1)$ & $-1(1)$ \\
\hline$C(7)$ & $30(1)$ & $29(1)$ & $24(1)$ & $-2(1)$ & $10(1)$ & $1(1)$ \\
\hline C ( 8) & $27(1)$ & $28(1)$ & $24(1)$ & $2(1)$ & $4(1)$ & $0(1)$ \\
\hline C (9) & $21(1)$ & $26(1)$ & $29(1)$ & $5(1)$ & $7(1)$ & $2(1)$ \\
\hline$C(10)$ & $25(1)$ & 21 (1) & $27(1)$ & $1(1)$ & $10(1)$ & $-1(1)$ \\
\hline$C(11)$ & $25(1)$ & 32 (1) & 25 (1) & $-1(1)$ & 11 (1) & $1(1)$ \\
\hline$C(12)$ & $26(1)$ & $42(1)$ & $37(1)$ & $-4(1)$ & $13(1)$ & $-5(1)$ \\
\hline$C(13)$ & $36(1)$ & $35(1)$ & 41 (1) & $1(1)$ & $20(1)$ & $10(1)$ \\
\hline$C(14)$ & $32(1)$ & $58(2)$ & 31 (1) & $2(1)$ & $17(1)$ & $2(1)$ \\
\hline$C(15)$ & 32 (1) & $43(1)$ & $23(1)$ & $1(1)$ & $2(1)$ & $2(1)$ \\
\hline$C(16)$ & $101(4)$ & $63(3)$ & $48(2)$ & $-30(2)$ & $-27(2)$ & $31(3)$ \\
\hline$C(17)$ & $62(2)$ & $138(5)$ & $32(2)$ & $31(3)$ & $4(2)$ & $-24(3)$ \\
\hline$C(18)$ & $36(2)$ & $99(4)$ & $32(2)$ & $3(2)$ & $-1(2)$ & $20(2)$ \\
\hline$C(16 A)$ & $101(4)$ & $63(3)$ & $48(2)$ & $-30(2)$ & $-27(2)$ & 31 (3) \\
\hline$C(17 A)$ & $62(2)$ & $138(5)$ & $32(2)$ & $31(3)$ & $4(2)$ & $-24(3)$ \\
\hline$C(18 A)$ & $36(2)$ & $99(4)$ & $32(2)$ & $3(2)$ & $-1(2)$ & $20(2)$ \\
\hline$C(19)$ & $25(1)$ & $32(1)$ & $30(1)$ & $1(1)$ & $12(1)$ & $4(1)$ \\
\hline$C(20)$ & $40(1)$ & $53(2)$ & $39(1)$ & $4(1)$ & $20(1)$ & $18(1)$ \\
\hline$C(21)$ & $35(1)$ & $31(1)$ & $38(1)$ & $-6(1)$ & $17(1)$ & $2(1)$ \\
\hline$C(22)$ & $30(1)$ & $47(1)$ & $36(1)$ & $1(1)$ & $16(1)$ & $-3(1)$ \\
\hline$C(23)$ & $59(2)$ & $59(2)$ & $45(2)$ & $-7(1)$ & $16(1)$ & $-5(2)$ \\
\hline C (24) & $77(2)$ & $56(2)$ & $37(1)$ & $1(1)$ & $23(2)$ & $-13(2)$ \\
\hline$C(25)$ & $67(2)$ & $56(2)$ & $69(2)$ & $-16(2)$ & $44(2)$ & $-14(2)$ \\
\hline$C(26)$ & $59(2)$ & $47(2)$ & $109(3)$ & $4(2)$ & $38(2)$ & $7(2)$ \\
\hline C (27) & $78(2)$ & $37(2)$ & $98(3)$ & $11(2)$ & $48(2)$ & $1(2)$ \\
\hline C (28) & $58(2)$ & $49(2)$ & $69(2)$ & $-12(2)$ & $33(2)$ & $-14(1)$ \\
\hline
\end{tabular}

The anisotropic displacement factor exponent takes the form $2 \mathrm{pi}^{\wedge} 2\left[\mathrm{~h}^{\wedge} 2 \mathrm{a} * \wedge 2 \mathrm{U}(11)+\ldots+2 \mathrm{hka} * \mathrm{~b} * \mathrm{U}(12)\right]$ 
Table S1-5. Hydrogen Coordinates (A $\times 10^{\wedge} 4$ ) and equivalent isotropic displacement parameters $\left(A^{\wedge} 2 \times 10^{\wedge} 3\right)$ for $9^{\prime}$

\begin{tabular}{|c|c|c|c|c|}
\hline atom & $\mathrm{x}$ & Y & z & $\mathrm{U}(\mathrm{eq})$ \\
\hline $\mathrm{H}(2 \mathrm{~A})$ & 2406 & -916 & 3935 & 31 \\
\hline $\mathrm{H}(2 \mathrm{~B})$ & 2279 & -1117 & 4675 & 31 \\
\hline $\mathrm{H}(3 \mathrm{~A})$ & 1481 & -3062 & 3510 & 54 \\
\hline $\mathrm{H}(3 \mathrm{~B})$ & 1965 & -3124.0002 & 4192 & 54 \\
\hline $\mathrm{H}(3 \mathrm{C})$ & 1829 & -3341 & 3207 & 54 \\
\hline $\mathrm{H}(4 \mathrm{~A})$ & 1280 & -1955 & 1883 & 55 \\
\hline $\mathrm{H}(4 \mathrm{~B})$ & 988 & -662 & 1746 & 55 \\
\hline $\mathrm{H}(4 \mathrm{C})$ & 995 & -1864 & 2356 & 55 \\
\hline $\mathrm{H}(7)$ & 1235 & 1414 & 133 & 34 \\
\hline $\mathrm{H}(9)$ & 346 & 2775 & 702 & 32 \\
\hline $\mathrm{H}(12 \mathrm{~A})$ & 2152 & 2311 & 2769 & 53 \\
\hline $\mathrm{H}(12 \mathrm{~B})$ & 2089 & 3301 & 2007 & 53 \\
\hline $\mathrm{H}(12 \mathrm{C})$ & 2472 & 2258 & 2377 & 53 \\
\hline $\mathrm{H}(13 \mathrm{~A})$ & 2333 & -248 & 1929 & 54 \\
\hline $\mathrm{H}(13 \mathrm{~B})$ & 1861 & -770 & 1582 & 54 \\
\hline $\mathrm{H}(13 \mathrm{C})$ & 2109 & -181.0000 & 2524 & 54 \\
\hline $\mathrm{H}(14 \mathrm{~A})$ & 1846 & 2398 & 552 & 59 \\
\hline $\mathrm{H}(14 \mathrm{~B})$ & 1824 & 794 & 412 & 59 \\
\hline $\mathrm{H}(14 \mathrm{C})$ & 2265 & 1513 & 979 & 59 \\
\hline $\mathrm{H}(16 \mathrm{~A})$ & 279 & -1 & -696 & 142 \\
\hline $\mathrm{H}(16 \mathrm{~B})$ & 644 & 275 & -952.9999 & 142 \\
\hline $\mathrm{H}(16 \mathrm{C})$ & 167 & 586 & -1615.0001 & 142 \\
\hline $\mathrm{H}(17 \mathrm{~A})$ & 837 & 2658 & -1113 & 127 \\
\hline $\mathrm{H}(17 \mathrm{~B})$ & 629 & 3881 & -850 & 127 \\
\hline $\mathrm{H}(17 \mathrm{C})$ & 364 & 3098 & -1726 & 127 \\
\hline $\mathrm{H}(18 \mathrm{~A})$ & -201 & 2551 & -1462 & 93 \\
\hline $\mathrm{H}(18 \mathrm{~B})$ & 12 & 3435 & -613 & 93 \\
\hline $\mathrm{H}(18 \mathrm{C})$ & -135 & 1918 & -581 & 93 \\
\hline $\mathrm{H}(16 \mathrm{D})$ & 221 & -2 & -1003 & 142 \\
\hline $\mathrm{H}(16 \mathrm{E})$ & -160 & 1025 & -1242 & 142 \\
\hline $\mathrm{H}(16 \mathrm{~F})$ & 121 & 577 & -277 & 142 \\
\hline $\mathrm{H}(17 \mathrm{D})$ & 823 & 845 & -1022 & 127 \\
\hline $\mathrm{H}(17 \mathrm{E})$ & 829 & 2422 & -1218 & 127 \\
\hline $\mathrm{H}(17 \mathrm{~F})$ & 441 & 1506 & -1832 & 127 \\
\hline $\mathrm{H}(18 \mathrm{D})$ & 2 & 3195 & -1564 & 93 \\
\hline $\mathrm{H}(18 \mathrm{E})$ & 375 & 3996 & -810 & 93 \\
\hline $\mathrm{H}(18 \mathrm{~F})$ & 10 & 3324 & -660 & 93 \\
\hline $\mathrm{H}(20 \mathrm{~A})$ & 303 & 4422 & 2299 & 65 \\
\hline $\mathrm{H}(20 \mathrm{~B})$ & 128 & 3465 & 1481 & 65 \\
\hline $\mathrm{H}(20 \mathrm{C})$ & 452 & 4650 & 1588 & 65 \\
\hline $\mathrm{H}(21 \mathrm{~A})$ & 1318 & 3247 & 3419 & 51 \\
\hline $\mathrm{H}(21 \mathrm{~B})$ & 973 & 4292 & 3394 & 51 \\
\hline $\mathrm{H}(21 \mathrm{C})$ & 1187 & 4518 & 2790 & 51 \\
\hline $\mathrm{H}(22 \mathrm{~A})$ & 811 & 1260 & 3087 & 56 \\
\hline $\mathrm{H}(22 \mathrm{~B})$ & 355 & 1367 & 2292 & 56 \\
\hline $\mathrm{H}(22 \mathrm{C})$ & 474 & 2293 & 3110 & 56 \\
\hline
\end{tabular}


Figure S2: Molecular structure of $\mathbf{1 0}$

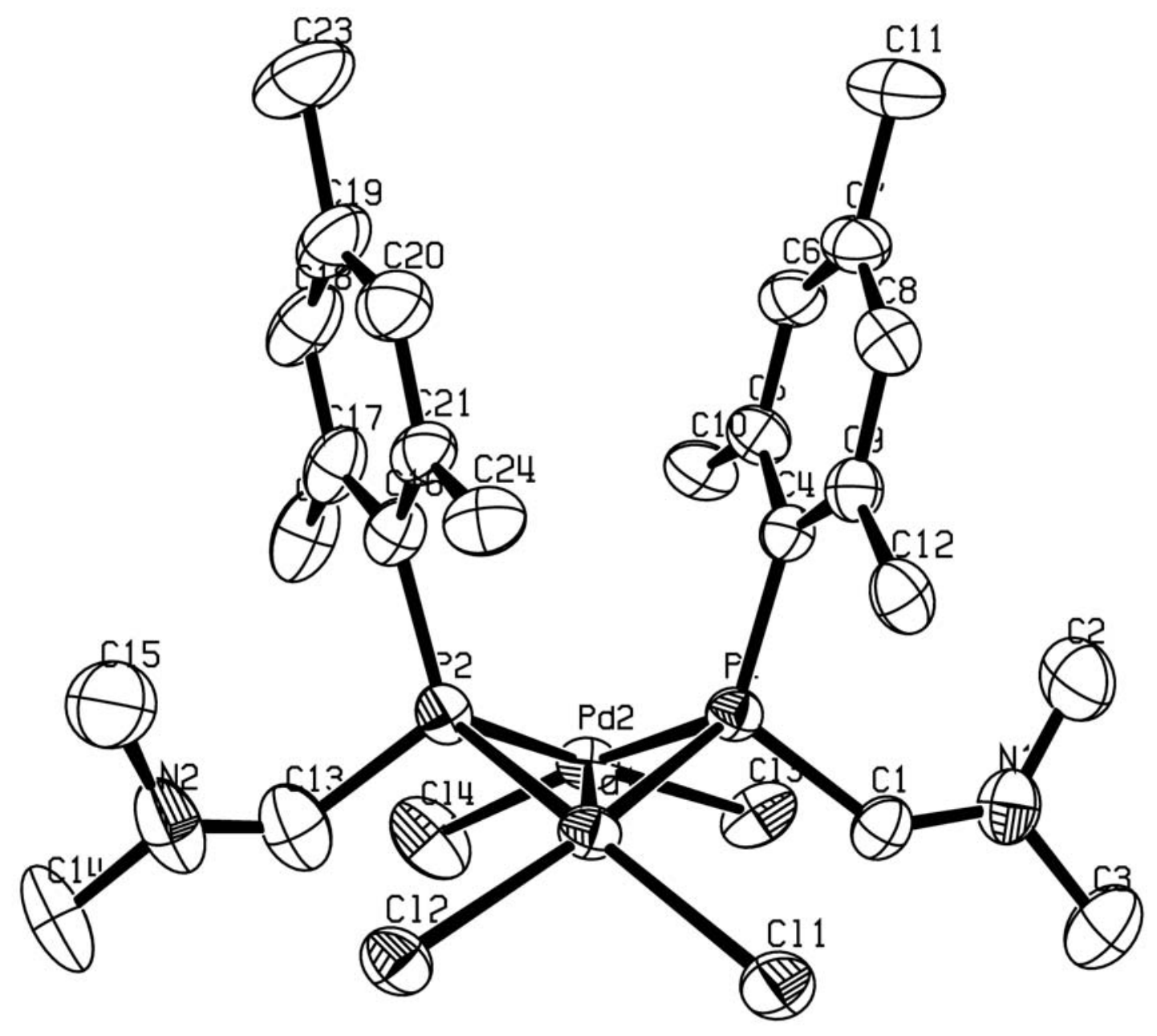


Compound

Molecular formula

Molecular weight

Crystal habit

Crystal dimensions(mm)

Crystal system

Space group

$\mathrm{a}(\AA)$
$\mathrm{b}(\AA)$
$\mathrm{c}(\AA)$
$\alpha\left(^{\circ}\right)$
$\beta\left(^{\circ}\right)$
$\gamma\left({ }^{\circ}\right)$
$\mathrm{V}\left(\AA^{3}\right)$
$\mathrm{Z}$

$\mathrm{d}\left(\mathrm{g}-\mathrm{cm}^{-3}\right)$

$\mathrm{F}(000)$

$\mu\left(\mathrm{cm}^{-1}\right)$

Absorption corrections

Diffractometer

$\mathrm{X}$-ray source

$\lambda(\AA)$

Monochromator

$\mathrm{T}(\mathrm{K})$

Scan mode

Maximum $\theta$

HKL ranges

Reflections measured

Unique data

Rint

Reflections used

Criterion

Refinement type

Hydrogen atoms

Parameters refined

Reflections / parameter

wR2

R1

Weights $a, b$

GoF

difference peak / hole (e $\left.\AA^{-3}\right)$ pdpalc

$\mathrm{C}_{24} \mathrm{H}_{36} \mathrm{Cl}_{4} \mathrm{~N}_{2} \mathrm{P}_{2} \mathrm{Pd}_{2}, 3.5\left(\mathrm{CHCl}_{3}\right)$

1186.88

magenta plate

$0.18 \times 0.12 \times 0.12$

monoclinic

$\mathrm{P} 21 / \mathrm{n}$

$12.1510(10)$

$16.8030(10)$

$22.6970(10)$

90.00

$100.8900(10)$

90.00

$4550.7(5)$

4

1.732

2348

1.736

multi-scan ; 0.7452 min, 0.8187 max

KappaCCD

$\mathrm{MoK} \alpha$

0.71069

graphite

150.0(1)

phi and omega scans

27.48

$-1215 ;-2119 ;-2922$

40629

10412

0.0626

7767

I $>2 \sigma \mathrm{I})$

Fsqd

mixed

426

18

0.1970

0.0650

$0.1045 ; 16.736$

1.070

4.927(0.206) / - $1.775(0.206)$

Note: Poorly resolved structure due to large mosaicity. Unresolved disorderis apparent. Large unexplained residue near $\mathrm{C} 15$. A chloroform moleculelocated near a symmetry center was incompletely resolved and accountedfor using the Platon SQUEEZE function. 
Table S2-2. Atomic Coordinates (A x 10^4) and equivalent isotropic displacement parameters $\left(A^{\wedge} 2 \times 10^{\wedge} 3\right)$ for 10

\begin{tabular}{|c|c|c|c|c|}
\hline atom & $\mathrm{x}$ & $\mathrm{Y}$ & $\mathrm{z}$ & $\mathrm{U}(\mathrm{eq})$ \\
\hline $\mathrm{Pd}(1)$ & $3569(1)$ & $2248(1)$ & $2290(1)$ & $29(1)$ \\
\hline $\mathrm{Pd}(2)$ & $6090(1)$ & $2288(1)$ & $2078(1)$ & 31 (1) \\
\hline $\mathrm{Cl}(1)$ & $2958(1)$ & $3103(1)$ & $2990(1)$ & $44(1)$ \\
\hline $\mathrm{Cl}(2)$ & $2667(1)$ & $1137(1)$ & $2634(1)$ & $39(1)$ \\
\hline$P(1)$ & $4667(1)$ & $3163(1)$ & $1935(1)$ & $28(1)$ \\
\hline$P(2)$ & $4553(1)$ & $1535(1)$ & $1728(1)$ & $31(1)$ \\
\hline$N(1)$ & $5178(5)$ & $4638(3)$ & $2534(3)$ & $43(1)$ \\
\hline $\mathrm{N}(2)$ & $4456(6)$ & $-89(4)$ & $2004(4)$ & $65(2)$ \\
\hline$C(1)$ & $5029(5)$ & $3891(4)$ & $2529(3)$ & $35(1)$ \\
\hline$C(2)$ & $5064(8)$ & $5153(5)$ & $2037(4)$ & $63(2)$ \\
\hline$C(3)$ & $5450(10)$ & $5066(6)$ & $3121(4)$ & $72(3)$ \\
\hline$C(4)$ & $4192(5)$ & $3622(3)$ & $1204(2)$ & 31 (1) \\
\hline$C(5)$ & $4882(5)$ & $3584(4)$ & $759(3)$ & $36(1)$ \\
\hline$C(6)$ & 4445 (5) & $3898(4)$ & $199(3)$ & $38(1)$ \\
\hline$C(7)$ & $3404(5)$ & $4251(4)$ & $50(3)$ & $40(1)$ \\
\hline$C(8)$ & $2755(5)$ & $4285(4)$ & $495(3)$ & $36(1)$ \\
\hline$C(9)$ & $3141(5)$ & $3982(3)$ & $1073(3)$ & $32(1)$ \\
\hline$C(10)$ & $6042(5)$ & $3248(4)$ & $857(3)$ & $43(2)$ \\
\hline$C(11)$ & $2998(7)$ & $4599(5)$ & $-558(3)$ & $57(2)$ \\
\hline$C(12)$ & $2395(5)$ & $4093(4)$ & $1531(3)$ & $38(1)$ \\
\hline$C(13)$ & $4789(6)$ & $589(5)$ & $2107(4)$ & $55(2)$ \\
\hline$C(14)$ & $4728(7)$ & $-821(5)$ & $2323(5)$ & $68(2)$ \\
\hline$C(15)$ & $3470(10)$ & $-235(7)$ & $1446(5)$ & $104(4)$ \\
\hline$C(16)$ & $4081(5)$ & $1438(4)$ & $924(3)$ & $37(1)$ \\
\hline$C(17)$ & $4837(6)$ & $1130(4)$ & $583(3)$ & $45(2)$ \\
\hline C (18) & $4502(7)$ & $1094(5)$ & $-42(3)$ & $57(2)$ \\
\hline C (19) & $3453(8)$ & $1373(5)$ & $-332(3)$ & $58(2)$ \\
\hline$C(20)$ & $2738(7)$ & $1670(5)$ & $13(3)$ & $51(2)$ \\
\hline$C(21)$ & $3009(5)$ & $1694(4)$ & $633(3)$ & $38(1)$ \\
\hline C (22) & $5982(6)$ & $837(5)$ & $850(4)$ & $60(2)$ \\
\hline$C(23)$ & $3100(10)$ & $1360(7)$ & $-1021(4)$ & $80(3)$ \\
\hline$C(24)$ & $2099(5)$ & $1979(4)$ & $960(3)$ & $42(2)$ \\
\hline$C(25)$ & $9056(6)$ & $2531(4)$ & $1469(3)$ & 41 (1) \\
\hline$C(26)$ & $6361(8)$ & $2910(5)$ & $-1138(4)$ & $59(2)$ \\
\hline$C(27)$ & $9734(8)$ & $3426(6)$ & $-1031(4)$ & $70(2)$ \\
\hline $\mathrm{Cl}(3)$ & $7416(1)$ & $3268(1)$ & $2476(1)$ & $47(1)$ \\
\hline Cl (4) & $7378(2)$ & $1247(1)$ & $2331(1)$ & $61(1)$ \\
\hline $\mathrm{Cl}(5)$ & $9147(2)$ & $3475(1)$ & $1136(1)$ & $60(1)$ \\
\hline Cl (6) & $10300(1)$ & $2311(1)$ & $1963(1)$ & $47(1)$ \\
\hline $\mathrm{Cl}(7)$ & $8762(2)$ & $1805(1)$ & $903(1)$ & $57(1)$ \\
\hline $\mathrm{Cl}(8)$ & $6742(2)$ & $3861(1)$ & $-831(1)$ & $54(1)$ \\
\hline $\mathrm{Cl}(9)$ & $6691(3)$ & $2180(1)$ & $-574(1)$ & $85(1)$ \\
\hline $\mathrm{Cl}(10)$ & $4942(2)$ & $2877(2)$ & $-1458(1)$ & $77(1)$ \\
\hline $\mathrm{Cl}(11)$ & $9721(4)$ & $2939(5)$ & $-375(2)$ & $196(3)$ \\
\hline $\mathrm{Cl}(12)$ & $9889(3)$ & $4435(3)$ & $-907(3)$ & $183(3)$ \\
\hline $\mathrm{Cl}(13)$ & $10827(2)$ & $3041(2)$ & $-1365(1)$ & $80(1)$ \\
\hline
\end{tabular}

$\mathrm{U}($ eq) is defined as $1 / 3$ the trace of the Uij tensor. 
Table S2-3. Bond lengths (A) and angles (deg) for 10

$\begin{array}{llll}\mathrm{Pd}(1)-\mathrm{P}(2) & 2.251(2) & \mathrm{Pd}(1)-\mathrm{P}(1) & 2.279(2) \\ \mathrm{Pd}(1)-\mathrm{Cl}(1) & 2.363(2) & \mathrm{Pd}(1)-\mathrm{Cl}(2) & 2.372(2) \\ \mathrm{Pd}(1)-\mathrm{Pd}(2) & 3.1896(6 & \mathrm{Pd}(2)-\mathrm{P}(1) & 2.246(2) \\ \mathrm{Pd}(2)-\mathrm{P}(2) & 2.272(2) & \mathrm{Pd}(2)-\mathrm{Cl}(4) & 2.345(2) \\ \mathrm{Pd}(2)-\mathrm{Cl}(3) & 2.361(2) & \mathrm{P}(1)-\mathrm{C}(1) & 1.811(6) \\ \mathrm{P}(1)-\mathrm{C}(4) & 1.823(6) & \mathrm{P}(2)-\mathrm{C}(13) & 1.804(8) \\ \mathrm{P}(2)-\mathrm{C}(16) & 1.814(6) & \mathrm{N}(1)-\mathrm{C}(1) & 1.269(8) \\ \mathrm{N}(1)-\mathrm{C}(2) & 1.41(1) & \mathrm{N}(1)-\mathrm{C}(3) & 1.49(1) \\ \mathrm{N}(2)-\mathrm{C}(13) & 1.22(1) & \mathrm{N}(2)-\mathrm{C}(14) & 1.43(1) \\ \mathrm{N}(2)-\mathrm{C}(15) & 1.59(1) & \mathrm{C}(4)-\mathrm{C}(9) & 1.393(8) \\ \mathrm{C}(4)-\mathrm{C}(5) & 1.431(8) & \mathrm{C}(5)-\mathrm{C}(6) & 1.385(8) \\ \mathrm{C}(5)-\mathrm{C}(10) & 1.495(9) & \mathrm{C}(6)-\mathrm{C}(7) & 1.380(9) \\ \mathrm{C}(7)-\mathrm{C}(8) & 1.396(9) & \mathrm{C}(7)-\mathrm{C}(11) & 1.494(9) \\ \mathrm{C}(8)-\mathrm{C}(9) & 1.403(8) & \mathrm{C}(9)-\mathrm{C}(12) & 1.514(8) \\ \mathrm{C}(16)-\mathrm{C}(17) & 1.407(9) & \mathrm{C}(16)-\mathrm{C}(21) & 1.412(9) \\ \mathrm{C}(17)-\mathrm{C}(18) & 1.40(1) & \mathrm{C}(17)-\mathrm{C}(22) & 1.49(1) \\ \mathrm{C}(18)-\mathrm{C}(19) & 1.40(1) & \mathrm{C}(19)-\mathrm{C}(20) & 1.37(1) \\ \mathrm{C}(19)-\mathrm{C}(23) & 1.54(1) & \mathrm{C}(20)-\mathrm{C}(21) & 1.384(9) \\ \mathrm{C}(21)-\mathrm{C}(24) & 1.520(9) & \mathrm{C}(25)-\mathrm{Cl}(6) & 1.743(7) \\ \mathrm{C}(25)-\mathrm{Cl}(7) & 1.760(7) & \mathrm{C}(25)-\mathrm{Cl}(5) & 1.770(7) \\ \mathrm{C}(26)-\mathrm{Cl}(10) & 1.74(1) & \mathrm{C}(26)-\mathrm{Cl}(9) & 1.763(9) \\ \mathrm{C}(26)-\mathrm{Cl}(8) & 1.771(8) & \mathrm{C}(27)-\mathrm{Cl}(11) & 1.70(1) \\ \mathrm{C}(27)-\mathrm{Cl}(12) & 1.72(1) & \mathrm{C}(27)-\mathrm{Cl}(13) & 1.77(1)\end{array}$

$\mathrm{P}(2)-\mathrm{Pd}(1)-\mathrm{P}(1)$
$\mathrm{P}(1)-\mathrm{Pd}(1)-\mathrm{C}(1)$
$\mathrm{P}(1)-\mathrm{Pd}(1)-\mathrm{C}(1)$
$\mathrm{P}(2)-\mathrm{Pd}(1)-\mathrm{Pd}(2)$
$\mathrm{C} 1(1)-\mathrm{Pd}(1)-\mathrm{Pd}(2)$
$\mathrm{P}(1)-\mathrm{Pd}(2)-\mathrm{P}(2)$
$\mathrm{P}(2)-\mathrm{Pd}(2)-\mathrm{C} 1(4)$
$\mathrm{P}(2)-\mathrm{Pd}(2)-\mathrm{C}(1)$
$\mathrm{P}(1)-\mathrm{P}(2)-\mathrm{Pd}(1)$
$\mathrm{C} 1(4)-\mathrm{Pd}(2)-\mathrm{Pd}(1)$
$\mathrm{C}(1)-\mathrm{P}(1)-\mathrm{C}(4)$
$\mathrm{C}(4)-\mathrm{P}(1)-\mathrm{Pd}(2)$
$\mathrm{C}(4)-\mathrm{P}(1)-\mathrm{Pd}(1)$
$\mathrm{C}(13)-\mathrm{P}(2)-\mathrm{C}(16)$
$\mathrm{C}(16)-\mathrm{P}(2)-\mathrm{Pd}(1)$
$\mathrm{C}(16)-\mathrm{P}(2)-\mathrm{Pd}(2)$
$\mathrm{C}(1)-\mathrm{N}(1)-\mathrm{C}(2)$
$\mathrm{C}(2)-\mathrm{N}(1)-\mathrm{C}(3)$
$\mathrm{C}(13)-\mathrm{N}(2)-\mathrm{C}(15)$
$\mathrm{N}(1)-\mathrm{C}(1)-\mathrm{P}(1)$
$\mathrm{C}(9)-\mathrm{C}(4)-\mathrm{P}(1)$
$\mathrm{C}(6)-\mathrm{C}(5)-\mathrm{C}(4)$
$\mathrm{C}(4)-\mathrm{C}(5)-\mathrm{C}(10)$
$\mathrm{C}(6)-\mathrm{C}(7)-\mathrm{C}(8)$
$\mathrm{C}(8)-\mathrm{C}(7)-\mathrm{C}(11)$
$\mathrm{C}(4)-\mathrm{C}(9)-\mathrm{C}(8)$
$\mathrm{C}(8)-\mathrm{C}(9)-\mathrm{C}(12)$
$\mathrm{C}(17)-\mathrm{C}(16)-\mathrm{C}(21)$
$\mathrm{C}(21)-\mathrm{C}(16)-\mathrm{P}(2)$
$\mathrm{C}(18)-\mathrm{C}(17)-\mathrm{C}(22)$
$\mathrm{C}(19)-\mathrm{C}(18)-\mathrm{C}(17)$
$\mathrm{C}(20)-\mathrm{C}(19)-\mathrm{C}(23)$
$\mathrm{C}(19)-\mathrm{C}(20)-\mathrm{C}(21)$
$\mathrm{C}(20)-\mathrm{C}(21)-\mathrm{C}(24)$
$\mathrm{C} 1(6)-\mathrm{C}(25)-\mathrm{C} 1(7)$
$\mathrm{C} 1(7)-\mathrm{C}(25)-\mathrm{C} 1(5)$
$\mathrm{C} 1(10)-\mathrm{C}(26)-\mathrm{C}(8)$
$\mathrm{C} 1(11)-\mathrm{C}(27)-\mathrm{C} 1(12)$
$\mathrm{C} 1(12)-\mathrm{C}(27)-\mathrm{Cl}(13)$

$75.54(5)$

$96.12(6)$

$170.14(6)$

$45.41(4)$

$121.40(5)$

$75.77(5)$

$97.89(7)$

$168.23(6)$

$45.61(4)$

$124.30(5)$

$112.5(3)$

$120.7(2)$

$120.1(2)$

$113.1(4)$

$121.1(2)$

$119.0(2)$

$127.4(6)$

$113.0(6)$

$118.1(8)$

$133.6(5)$

$119.8(4)$

$117.2(5)$

$125.0(5)$

$117.2(5)$

$121.3(6)$

$119.1(5)$

$117.3(5)$

$119.7(6)$

$122.1(5)$

$117.9(7)$

$121.8(7)$

$120(1)$

$122.5(8)$

$116.8(6)$

$110.7(4)$

$109.3(4)$

$111.0(5)$

$110.5(6)$

$111.2(6)$
$\mathrm{P}(2)-\mathrm{Pd}(1)-\mathrm{Cl}(1)$

$\mathrm{P}(2)-\mathrm{Pd}(1)-\mathrm{Cl}(2)$

$\mathrm{Cl}(1)-\mathrm{Pd}(1)-\mathrm{Cl}(2)$

$\mathrm{P}(1)-\mathrm{Pd}(1)-\mathrm{Pd}(2)$

$\mathrm{Cl}(2)-\mathrm{Pd}(1)-\mathrm{Pd}(2)$

$P(1)-P d(2)-C l(4)$

$\mathrm{P}(1)-\mathrm{Pd}(2)-\mathrm{Cl}(3)$

$\mathrm{Cl}(4)-\mathrm{Pd}(2)-\mathrm{Cl}(3)$

$P(2)-P d(2)-P d(1)$

$\mathrm{Cl}(3)-\mathrm{Pd}(2)-\mathrm{Pd}(1)$

$\mathrm{C}(1)-\mathrm{P}(1)-\mathrm{Pd}(2)$

$\mathrm{C}(1)-\mathrm{P}(1)-\mathrm{Pd}(1)$

$\mathrm{Pd}(2)-\mathrm{P}(1)-\mathrm{Pd}(1)$

$C(13)-P(2)-P d(1)$

$C(13)-P(2)-P d(2)$

$P d(1)-P(2)-P d(2)$

$\mathrm{C}(1)-\mathrm{N}(1)-\mathrm{C}(3)$

$\mathrm{C}(13)-\mathrm{N}(2)-\mathrm{C}(14)$

$\mathrm{C}(14)-\mathrm{N}(2)-\mathrm{C}(15)$

$C(9)-C(4)-C(5)$

$\mathrm{C}(5)-\mathrm{C}(4)-\mathrm{P}(1)$

$C(6)-C(5)-C(10)$

$C(7)-C(6)-C(5)$

$C(6)-C(7)-C(11)$

$C(7)-C(8)-C(9)$

$C(4)-C(9)-C(12)$

$\mathrm{N}(2)-\mathrm{C}(13)-\mathrm{P}(2)$

$\mathrm{C}(17)-\mathrm{C}(16)-\mathrm{P}(2)$

$\mathrm{C}(18)-\mathrm{C}(17)-\mathrm{C}(16)$

$C(16)-C(17)-C(22)$

$\mathrm{C}(20)-\mathrm{C}(19)-\mathrm{C}(18)$

$\mathrm{C}(18)-\mathrm{C}(19)-\mathrm{C}(23)$

$C(20)-C(21)-C(16)$

$C(16)-C(21)-C(24)$

$\mathrm{Cl}(6)-\mathrm{C}(25)-\mathrm{Cl}(5)$

$\mathrm{Cl}(10)-\mathrm{C}(26)-\mathrm{Cl}(9)$

$\mathrm{Cl}(9)-\mathrm{C}(26)-\mathrm{Cl}(8)$

$\mathrm{Cl}(11)-\mathrm{C}(27)-\mathrm{Cl}(13)$
$166.49(6)$

$95.47(6)$

$91.92(6)$

$44.77(4)$

$125.66(4)$

$169.64(7)$

$92.86(6)$

$92.90(7)$

$44.88(4)$

$124.24(5)$

$105.2(2)$

$105.6(2)$

$89.62(5)$

$104.6(3)$

$106.1(3)$

$89.71(6)$

$119.5(7)$

$132(1)$

$109.6(7)$

$120.4(5)$

$119.8(4)$

$117.8(6)$

$124.3(6)$

$121.5(6)$

$121.8(6)$

$123.5(5)$

$135.7(8)$

$118.2(5)$

$118.6(7)$

$123.6(6)$

$118.2(7)$

$121.7(8)$

$119.2(6)$

$124.0(5)$

$110.4(4)$

$110.7(5)$

$109.6(5)$

$109.3(6)$ 
Table S2-4. Anisotropic displacement parameters ( $A^{\wedge} 2 \times 10^{\wedge} 3$ ) for 10

\begin{tabular}{|c|c|c|c|c|c|c|}
\hline atom & U11 & U22 & U33 & U23 & U13 & U12 \\
\hline $\mathrm{Pd}(1)$ & $26(1)$ & $34(1)$ & $28(1)$ & $3(1)$ & $9(1)$ & $2(1)$ \\
\hline $\mathrm{Pd}(2)$ & $23(1)$ & $40(1)$ & $30(1)$ & $4(1)$ & $5(1)$ & $3(1)$ \\
\hline $\mathrm{Cl}(1)$ & $49(1)$ & $47(1)$ & $42(1)$ & $2(1)$ & $24(1)$ & $7(1)$ \\
\hline $\mathrm{Cl}(2)$ & $37(1)$ & $40(1)$ & $42(1)$ & $6(1)$ & $15(1)$ & $-3(1)$ \\
\hline P (1) & $27(1)$ & 31 (1) & $28(1)$ & $1(1)$ & $8(1)$ & $1(1)$ \\
\hline$P(2)$ & $29(1)$ & $30(1)$ & $37(1)$ & $2(1)$ & 11 (1) & $2(1)$ \\
\hline $\mathrm{N}(1)$ & $37(3)$ & $39(3)$ & $53(3)$ & $-7(2)$ & $5(2)$ & $-7(2)$ \\
\hline $\mathrm{N}(2)$ & $78(5)$ & $37(3)$ & $97(5)$ & $12(3)$ & $61(4)$ & $11(3)$ \\
\hline$C(1)$ & $33(3)$ & $38(3)$ & $38(3)$ & $-6(2)$ & $13(2)$ & $-4(3)$ \\
\hline$C(2)$ & $66(5)$ & $48(4)$ & $68(5)$ & $1(4)$ & $-4(4)$ & $-18(4)$ \\
\hline$C(3)$ & $90(7)$ & $63(5)$ & $59(5)$ & $-23(4)$ & $1(4)$ & $-10(5)$ \\
\hline$C(4)$ & $35(3)$ & $29(3)$ & $30(3)$ & $-2(2)$ & $5(2)$ & $-4(2)$ \\
\hline$C(5)$ & $32(3)$ & $38(3)$ & $38(3)$ & $6(2)$ & $7(2)$ & $1(3)$ \\
\hline$C(6)$ & $41(3)$ & $43(3)$ & 31 (3) & $3(2)$ & $13(2)$ & $-7(3)$ \\
\hline$C(7)$ & $41(3)$ & $42(3)$ & $33(3)$ & $5(3)$ & $-3(2)$ & $-8(3)$ \\
\hline$C(8)$ & $30(3)$ & $36(3)$ & $43(3)$ & $4(2)$ & $5(2)$ & -1 (2) \\
\hline$C(9)$ & $29(3)$ & $29(3)$ & $36(3)$ & $-2(2)$ & $5(2)$ & $-3(2)$ \\
\hline$C(10)$ & $39(3)$ & $55(4)$ & $40(3)$ & $10(3)$ & $20(3)$ & $8(3)$ \\
\hline C (11) & $49(4)$ & $79(5)$ & $37(3)$ & $17(3)$ & $-4(3)$ & $-12(4)$ \\
\hline$C(12)$ & $32(3)$ & $34(3)$ & $50(3)$ & $5(3)$ & $13(3)$ & $9(2)$ \\
\hline C (13) & $48(4)$ & $45(4)$ & $78(5)$ & $11(4)$ & $25(4)$ & $5(3)$ \\
\hline C (14) & $47(4)$ & $37(4)$ & $119(7)$ & 31 ( 4) & $16(5)$ & $3(3)$ \\
\hline C (15) & $150(10)$ & $88(8)$ & $68(6)$ & $1(5)$ & $-5(7)$ & $-65(8)$ \\
\hline$C(16)$ & $35(3)$ & $39(3)$ & $41(3)$ & $-8(3)$ & $14(2)$ & $-6(3)$ \\
\hline C (17) & $41(4)$ & $45(4)$ & $54(4)$ & $-16(3)$ & $18(3)$ & $-11(3)$ \\
\hline C (18) & $57(5)$ & $69(5)$ & $55(4)$ & $-28(4)$ & $31(4)$ & $-24(4)$ \\
\hline C (19) & $80(6)$ & $59(5)$ & $41(4)$ & $-17(3)$ & $27(4)$ & $-20(4)$ \\
\hline$C(20)$ & $58(4)$ & $53(4)$ & $43(4)$ & $-2(3)$ & $8(3)$ & $-14(3)$ \\
\hline C (21) & $38(3)$ & $41(3)$ & $34(3)$ & $-4(2)$ & $5(2)$ & $-8(3)$ \\
\hline C (22) & $39(4)$ & $60(5)$ & $87(6)$ & $-31(4)$ & $29(4)$ & $-2(3)$ \\
\hline$C(23)$ & $100(7)$ & $107(8)$ & $39(4)$ & $-20(5)$ & $28(4)$ & $-38(6)$ \\
\hline C (24) & 30 (3) & $57(4)$ & $38(3)$ & $-1(3)$ & $2(2)$ & $-6(3)$ \\
\hline$C(25)$ & $35(3)$ & $48(4)$ & $40(3)$ & $-1(3)$ & $10(3)$ & $2(3)$ \\
\hline$C(26)$ & $85(6)$ & $46(4)$ & $57(4)$ & $-6(3)$ & $42(4)$ & $-3(4)$ \\
\hline C (27) & $60(5)$ & $98(7)$ & $49(4)$ & $-14(4)$ & $0(4)$ & $0(5)$ \\
\hline $\mathrm{Cl}(3)$ & $29(1)$ & $69(1)$ & $43(1)$ & $-15(1)$ & $6(1)$ & $-9(1)$ \\
\hline $\mathrm{Cl}(4)$ & $34(1)$ & $63(1)$ & $84(1)$ & $25(1)$ & $7(1)$ & $17(1)$ \\
\hline $\mathrm{Cl}(5)$ & $58(1)$ & $50(1)$ & $70(1)$ & $10(1)$ & 11 (1) & $1(1)$ \\
\hline $\mathrm{Cl}(6)$ & 37 (1) & 59 (1) & $43(1)$ & $-1(1)$ & $6(1)$ & $3(1)$ \\
\hline $\mathrm{Cl}(7)$ & $61(1)$ & $62(1)$ & $46(1)$ & $-8(1)$ & 7 (1) & $-14(1)$ \\
\hline $\mathrm{Cl}(8)$ & $65(1)$ & $50(1)$ & $52(1)$ & $-8(1)$ & $24(1)$ & $-11(1)$ \\
\hline Cl (9) & $136(3)$ & $51(1)$ & $80(2)$ & $5(1)$ & $47(2)$ & $-1(1)$ \\
\hline $\mathrm{Cl}(10)$ & $79(2)$ & $77(2)$ & $80(2)$ & $-19(1)$ & $28(1)$ & $-31(1)$ \\
\hline $\mathrm{Cl}$ (11) & $128(3)$ & $386(9)$ & $83(2)$ & $93(4)$ & $46(2)$ & $101(5)$ \\
\hline $\mathrm{Cl}$ (12) & $59(2)$ & $123(3)$ & $343(8)$ & $-102(4)$ & $-22(3)$ & $16(2)$ \\
\hline $\mathrm{Cl}(13)$ & $55(1)$ & $119(2)$ & $67(1)$ & $-25(1)$ & $15(1)$ & $-11(1)$ \\
\hline
\end{tabular}

The anisotropic displacement factor exponent takes the form $2 \mathrm{pi}^{\wedge} 2\left[\mathrm{~h}^{\wedge} 2 \mathrm{a} \star \wedge 2 \mathrm{U}(11)+\ldots+2 \mathrm{hka} * \mathrm{~b} * \mathrm{U}(12)\right]$ 
Table S2-5. Hydrogen Coordinates (A x 10^4) and equivalent isotropic displacement parameters $\left(A^{\wedge} 2 \times 10^{\wedge} 3\right)$ for 10

\begin{tabular}{|c|c|c|c|c|}
\hline atom & $\mathrm{x}$ & $\mathrm{Y}$ & z & $\mathrm{U}(\mathrm{eq})$ \\
\hline $\mathrm{H}(1)$ & 5129 & 3665 & 2919 & 43 \\
\hline $\mathrm{H}(2 \mathrm{~A})$ & 4947 & 4839 & 1667 & 95 \\
\hline $\mathrm{H}(2 \mathrm{~B})$ & 5745 & 5473 & 2064 & 95 \\
\hline $\mathrm{H}(2 \mathrm{C})$ & 4419 & 5504 & 2034 & 95 \\
\hline $\mathrm{H}(3 \mathrm{~A})$ & 5341 & 4705 & 3445 & 109 \\
\hline $\mathrm{H}(3 \mathrm{~B})$ & 4947 & 5525 & 3113 & 109 \\
\hline $\mathrm{H}(3 \mathrm{C})$ & 6226 & 5248 & 3189 & 109 \\
\hline $\mathrm{H}(6)$ & 4892 & 3869 & -102 & 45 \\
\hline $\mathrm{H}(8)$ & 2032 & 4521 & 403 & 44 \\
\hline $\mathrm{H}(10 \mathrm{~A})$ & 6362 & 3333 & 496 & 65 \\
\hline $\mathrm{H}(10 \mathrm{~B})$ & 6014 & 2676 & 938 & 65 \\
\hline $\mathrm{H}(10 \mathrm{C})$ & 6508 & 3515 & 1199 & 65 \\
\hline $\mathrm{H}(11 \mathrm{~A})$ & 3547 & 4985 & -649.0001 & 85 \\
\hline $\mathrm{H}(11 \mathrm{~B})$ & 2280 & 4868 & -565 & 85 \\
\hline $\mathrm{H}(11 \mathrm{C})$ & 2900 & 4174 & -859 & 85 \\
\hline $\mathrm{H}(12 \mathrm{~A})$ & 1608 & 4044 & 1333 & 57 \\
\hline $\mathrm{H}(12 \mathrm{~B})$ & 2523 & 4622 & 1713 & 57 \\
\hline $\mathrm{H}(12 \mathrm{C})$ & 2570 & 3685 & 1843 & 57 \\
\hline $\mathrm{H}(13)$ & 5299 & 631 & 2479 & 66 \\
\hline $\mathrm{H}(14 \mathrm{~A})$ & 5351 & -1080 & 2180 & 102 \\
\hline $\mathrm{H}(14 \mathrm{~B})$ & 4073 & -1173 & 2253 & 102 \\
\hline $\mathrm{H}(14 \mathrm{C})$ & 4945 & -710.9999 & 2753 & 102 \\
\hline $\mathrm{H}(15 \mathrm{~A})$ & 2748 & -102 & 1558 & 156 \\
\hline $\mathrm{H}(15 \mathrm{~B})$ & 3462 & -795 & 1324 & 156 \\
\hline $\mathrm{H}(15 \mathrm{C})$ & 3579 & 103 & 1110 & 156 \\
\hline $\mathrm{H}(18)$ & 5000 & 875 & -275 & 69 \\
\hline $\mathrm{H}(20)$ & 2028 & 1866 & -181.0000 & 62 \\
\hline $\mathrm{H}(22 \mathrm{~A})$ & 6330 & 603 & 534 & 89 \\
\hline $\mathrm{H}(22 \mathrm{~B})$ & 5928 & 432 & 1154 & 89 \\
\hline $\mathrm{H}(22 \mathrm{C})$ & 6440 & 1282 & 1037 & 89 \\
\hline $\mathrm{H}(23 \mathrm{~A})$ & 2416 & 1047 & -1135 & 120 \\
\hline$H(23 B)$ & 3703 & 1120 & -1196 & 120 \\
\hline $\mathrm{H}(23 \mathrm{C})$ & 2969 & 1905 & -1170 & 120 \\
\hline $\mathrm{H}(24 \mathrm{~A})$ & 1954 & 1568 & 1242 & 64 \\
\hline $\mathrm{H}(24 \mathrm{~B})$ & 1410 & 2084 & 668 & 64 \\
\hline $\mathrm{H}(24 \mathrm{C})$ & 2345 & 2468 & 1180 & 64 \\
\hline $\mathrm{H}(25)$ & 8430 & 2542 & 1698 & 49 \\
\hline $\mathrm{H}(26)$ & 6804 & 2798 & -1458 & 70 \\
\hline $\mathrm{H}(27)$ & 9004 & 3328 & -1307.9999 & 84 \\
\hline
\end{tabular}

